\title{
GENERALIZED PARTIAL RESPONSE TARGETS FOR PERPENDICULAR RECORDING
}

Piya Kovintavewat, Inci Ozgunes, ${ }^{*}$ Erozan Kurtas, ${ }^{*}$ John R. Barry and Steven W. McLaughlin

Georgia Institute of Technology, Atlanta, GA 30332

*Seagate Technology, Pittsburgh, PA 15203

\section{Introduction}

A new model for the transition response in perpendicular recording was proposed in [1], namely $g(t)=\operatorname{erf}\left(\sqrt{\ln 16} t / P W_{50}\right)$, where $\operatorname{erf}(\cdot)$ is the error function and $P W_{50}$ is the width of the derivative of $g(t)$ at half its maximum. We propose new generalized PR (GPR) targets based on this transition response and compare their performance.

\section{Channel Model}

We assume a linear binary-input $\{ \pm 1\}$ channel with bit period $T$ and impulse (dibit) response $g(t)-$ $g(t-T)$, equalized to a PR target (with integer coefficients) or a GPR target (with arbitrary coefficients). The additive noise before the equalizer is white and Gaussian with (two-sided) power spectral density $N_{0} / 2$. Because the impulse response contains a d.c. component, PR targets of the form $(1+D)^{n}$ are appropriate, where $D$ is the delay operator and $n$ is an integer. Following [2], we choose the GPR target so as to minimize mean-squared error, subject to a monic constraint.

\section{Results}

Fig. 1 compares the performance of different targets by plotting SNR requirement for BER $=10^{-4}$ as a function of the density $D_{u}=P W_{50} / T$, where SNR $=g(\infty)^{2} /\left(N_{0} /(2 T)\right)=$ $2 T / N_{0}$. The SNR requirement was found by simulations of the Viterbi detector, assuming an uncoded input sequence, a 7-th order Butterworth low-pass filter, and a 21-tap linear equalizer. For each $D_{u}$, the SNR used to design the target and its corresponding equalizer was chosen to minimize the SNR required to achieve the desired BER. From the figure we see that the GPR targets outperform the PR targets, especially at high recording densities.

Unlike longitudinal recording, where the worst-case error sequence was shown to be $\{2,-2,2\}$ [2], we have found that, for all targets, the worst-case error sequence for this

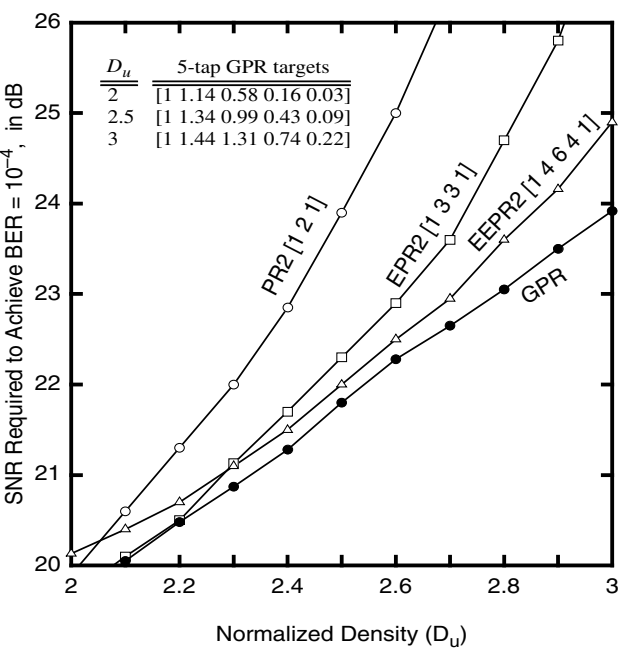

Fig. 1. Performance comparison. channel is $\{2,-2\}$, which corresponds to two consecutive transitions being shifted by one bit period. Performance can be further improved by designing and using codes that avoid this error sequence.

\section{References}

[1] Inci Ozgunes, Walter Eppler, and Erozan Kurtas, "Performance of Transforms used for Perpendicular Recording Systems," submitted to ICC 2002.

[2] Jaekyun Moon and Weining Zeng, "Equalization for Maximum-Likelihood Detectors," IEEE Trans. on Magnetics, vol. 31, no. 2, pp. 1083-1088, March 1995. 